\title{
Total diz protezi ve aseptik gevşeme
}

\author{
Total knee arthroplasty and aseptic loosening
}

\author{
Kasım Kılıçarslan, Enejd Veizi
}

Ankara Şehir Hastanesi, Ortopedi ve Travmatoloji Kliniği, Ankara

\begin{abstract}
Total diz protezi (TDP) cerrahisi tüm dünyada en sık uygulanan cerrahi işlemlerinden biridir. Her yıl yaklaşık 500.000 yeni cerrahi uygulanmakta olup, bu sayının 2030 yılına kadar $>1,2$ kat artacağı öngörülmektedir. En sık revizyon sebepleri arasında aseptik gevşeme bulunmaktadır. Avustralya ve İngiltere'nin ulusal kayıtlarına göre revizyon cerrahilerinin en sık sebebinin sırasıyla $\% 25,3$ ve $\% 32,7$ ile aseptik gevşemenin olduğu tespit edilmiştir. Enfeksiyöz sebeplerin dışında, protezin kemikten ayrılması anlamına gelen aseptik gevşemenin nedeni olarak birçok mekanizma sorumlu tutulmuştur. Başlangıçta implant-kemik arasındaki bağlantının bozulması ve buna bağlı implantın mikro-hareketiyle başlayan bu süreç, zamanla implantın çökmesine ve arayüzün aşınmasına sebep olur. Aşınan ara yüzeyden ortaya çıkan parçacıklar bir enflamatuvar süreci başlatır ve yaygın bir fagositoz gerçekleşir. Fagositoza uğrayan parçacıkların yanı sıra sağlam spongiyoz kemik de etkilenir ve lizise uğrar. Bu litik süreç zamanla implantın gevşemesine ve instabil hale gelmesine sebep olur. Erken tanı ve uygun tedavi, zamanla gelişebilecek komplikasyonları engellemek adına büyük öneme sahiptir.
\end{abstract}

Anahtar sözcükler: total diz protezi; aseptik gevşeme; aşınma; kemik lizisi
Total knee arthroplasty (TKA) is one of the most commonly performed surgical procedures worldwide. Every year approximately 500,000 new knees are implanted, and the numbers are expected to rise by 1.2 fold by 2030 . The most common cause for revision is aseptic loosening. According to the Australian and British National Arthroplasty Registers total knees fail by aseptic loosening by 25.3 and $32.7 \%$ respectively. Aseptic loosening is defined as disruption or impairment of the implant-bone contact surface in the absence of infection. This disruption allows micro-movements at the contact surface which can lead to implant subsidence and wear. The micro-particles resulting from the wear trigger an inflammatory response with widespread phagocytosis. The activated macrophages affect the cancellous bone as well leading to lysis. Because of this lytic process the implant will loosen and with time, become instable. Early diagnosis and adequate treatment are of paramount importance in order to avoid possible complications.

Key words: total knee arthroplasty, aseptic loosening; wear; bone lysis

\section{ETIYOLOJi}

Aseptik gevşeme, enfeksiyöz faktörlerin yokluğunda, kemik-implant veya kemik-çimento arasındaki bağlantının bozulmasına bağlı olarak meydana gelen instabiliteyi tarif etmek için kullanılan bir terimdir. Uzun süreli kullanıma bağlı olarak tüm implant çeşitlerinde değişik miktarlarda meydana gelebilir. Erken dönemde meydana gelmesi ise hastanın genç yaşta olması, implant tasarım eksiklikleri ve cerrahi hatalar ile ilişkilendirilmiştir. ${ }^{[1-3]}$

Başlangıçta implant-kemik arasındaki bağlantının bozulması ve buna bağı implantın mikro-hareketiyle başlayan bu süreç, zamanla implantın çökmesine ve ara yüzü oluşturan polietilen (PE) aşınmasına sebep olur. ${ }^{[4]}$

İletişim / Contact: Op. Dr. Enejd Veizi • E-posta / E-mail: dr.nad89@hotmail.com

ORCID iD: Kasım Kılıçarslan, 0000-0003-3852-4128 • Enejd Veizi, 0000-0002-1289-4959

Geliş / Received: 7 Temmuz 2021 • Kabul / Accepted: 24 Temmuz 2021 
Aşınma birçok şekilde olur:

Adheziv/abraziv aşınma: Aşınma kompresif güçlere bağlı olarak metalik komponent ve polietilen arasındaki yüzeylerin öğütülmesi sonucu meydana gelen kütle kaybına bağlıdır. Metalik implant, PE üzerinden 'rende' etkisi yapar ve daha büyük parçaları koparır. Zamana bağı tüm implantlarda meydana gelebilir ve aşırı kullanım süreci hızlandırır. Ortaya çıkan mikron büyüklükteki parçacıklar osteolizin tetiklenmesine sebep olur. En sık kalça ekleminde görülür. ${ }^{[5]}$

Üçüncü cisim aşınması: Eklem aralığında gezen cisimler (PE kalıntıları, serbest çimento veya kemik fragmanları vb.) tarafindan oluşturulan aşınma şekli.

Delaminasyon aşınması: Travma sonucu meydana gelen çatlamalar ve çukurlaşmaları tanımlar. Travmalar sonucu PE'nin şekli bozulur ve ortaya çıkan parçacıklar osteolize yol açar. Diz eklemi birçok eksene hareket edebiliyor olması sebebiyle delaminasyona en açık eklemdir.

Koroziv aşınmalar: Kimyasal aşınmaları ifade eder. ${ }^{[6,7]}$

Aşınma erken veya geç meydana gelebilir. Geç aşınma tüm implantlar için kaçınılmazken, erken aşınma genelde cerrahi hatalar, aşırı kullanım veya PE malzemesinden kaynaklanır. Bozuk dizilim ile yerleştirilen implantlar ara yüzeylere fazla stres bindirir ve delaminasyon kaçınılmaz olur. ${ }^{[8]}$ Eklem aralığının dar/sıkı olarak bırakılması, ekstansiyon aralığın geniş bırakılması, ince insert kullanılması ve protezin arka çapraz bağı kesen bir implantın olması erken aşınma riskini artırır. Ek olarak, çimentolama esnasında taşan çimento parçacıkların temizlenmemesi (özellikle femoral notch bölgesinde) erken aşınmayı tetikler. ${ }^{[8]}$

Hastaların aktivite düzeyi de aşınmayı etkilemektedir. Aktif hayat tarzı olan, morbid obez (Vücut Kitle İndeksi "VKi" >35) ve genç erkeklerde aşınma daha sık gözlemlenir. ${ }^{[1,9]}$

Az uyumlu veya aşırı uyumlu PE yüzeyleri ile düz PE yüzeylerine nazaran uygulanan stres miktarı daha fazla olduğundan dolayı aşınma daha sık görülür. Aşırı uyum, diz ekleminde rotasyonu engellediği için stresi artırırken; az uyumlu ve düz PE yüzeyler, fazla harekete izin verildiği için instabiliteye yatkınlık yaratır. Aşınma, PE kalınlığıyla da ilişkilidir. Altı milimetreden ince PE ara yüzeyleri aşınmaya daha yatkındır. Patellar komponentlerin çoğunlukla PE'den üretilmesi her zaman aşınmaya daha meyilli olmuştur. Metal arka yüzle desteklenen pateller implantlar aşınmaya daha yatkınken, tam PE ve geniş yüzeyli (sombrero şapkası gibi) olan patellar implantlar daha dayanıklıdır. ${ }^{[4-6]}$

Komponentlerin metal alaşımlarındaki farklılıklar da aşınmaya katkıda bulunur. Eskiden çok kullanılan titanyum alaşımlar, hızlı ve sık aşınmaya sebep oldukları için artık kullanılmamaktadır. Kobalt-krommolibden alaşımları son yıllarda yaygın bir şekilde kullanıma girmiştir ve titanyuma göre aşınma katsayıları önemli ölçüde düşüktür. En düşük aşınmaya sebep olan ise seramik komponentlerdir. Ancak sertliklerinden dolayı kırılmaya daha yatkındır ve cerrahi sonrası eklemde krepitasyona benzer sese neden olabilir. Tüm teknolojik gelişmelere rağmen diz potezi uygulamalarında sadece adhesiv aşınma azaltılabilmiştir. Daha sık görünen delaminasyon ise, gelişmelere rağmen halen sık görülmektedir. ${ }^{[6]}$

Son olarak PE, sterilizasyon yönteminden ve saklama koşullarından da etkilenen bir maddedir. En uygun sterilizasyon şekli etilen oksit (EO) gaz olup, saklanma süresinin 3-4 yılı geçmemesi gerekir. Gama ışınların veya oksijenin varlığında kimyasal özellikleri bozulmaya başladığından dolayı aşınmaya daha yatkın hale gelir. ${ }^{[10]}$

\section{PATOFIZYOLOJi}

Aşınan PE'den ortaya çıkan parçacıklar, makrofajlar tarafından tetiklenen bir enflamatuvar süreci başlatır ve yaygın bir fagositoz gerçekleşir. Parçacıkların boyutu ve konumu önemlidir. Büyük parçacıklar osteolize sebep olmazken, 1-100 $\mu \mathrm{m}$ arasında olan parçacıklar dev hücreler tarafindan fagosite edilir ve enflamatuvar reaksiyon başlatır. ${ }^{[11]}$ Osteolize sebep olmaları için bu parçacıkların spongiyoz kemikle temasa geçmeleri gerekir. Bu temas, çimentolu implantlar için çimentokemik arasındaki ilişkinin bozulmasıyla, çimentosuz implantlar için ise tespit vidaları ve kemiğin arasındaki bağlantının gevşemesiyle meydana gelir. ${ }^{[6]}$ Çimentonun kırılması veya yerleştirme esnasında spongiyoz kemiğin tam kaplanmaması osteoliz riskini artırı. Çimentosuz implantların tespiti için kullanılan vidaların yaklaşık \%16'sında uzun vadede osteoliz meydana geldiği bilinmektedir. ${ }^{[10,12]}$ Kemiklerin metafizinde, bazen fokal bazen de yaygın defektler oluşmaya başlar. Bu litik süreç zamanla implantın gevşemesine ve instabil hale gelmesine sebep olur.

Aseptik gevşemenin ana sebeplerinden biri, aşırı kullanımdır. Yaş ilerledikçe, aseptik gevşeme olasılığı da artar. Osteoporotik kemik, implantı destekleyecek gücünü kaybeder ve çökmesine izin verir. Bununla birlikte erkek cinsiyet ve genç yaştaki (<45 yaş) hastalar, fiziksel olarak daha aktif olmaları nedeniyle risk oluştururken, morbid obezite aseptik gevşemeye yardımcı olan başka bir sebeptir. ${ }^{[13]}$

Son yıllarda önemli gelişmeler kaydedilse de implant tasarım eksiklikleri hâlâ sık bir gevşeme sebebidir. Anatomik tasarımı olan, tibial stemi uzun olan, modüler olmayan ve minimal kısıtlamaya sebep olan 
implantların daha uzun ömürlü oldukları tespit edilmiştir. ${ }^{[9,14]}$ Kemik-implant ara yüzeyinde yüksek streslere neden olan kısıtlı implantlar, çimentosuz komponentler, modüler ve yüksek fleksiyon derecelerine izin veren tasarımlar gevşemeye daha müsaittir. ${ }^{[1]}$

Tam kaplama sağlamayan zayıf ve sığ çimentolama tekniği başka bir aseptik gevşeme sebebidir. Az miktarda çimento kullanılması spongiyoz kemiğin tam kaplanmamasına sebep olabilir. Bu olay cerrahi sonrası implantın mikro-hareketine izin verir ve osteolizisi tetikler. Çimentolamanın optimal derinliği tartışmalı olsa da son yayınlar bir trabeküler düzeyi katedecek şekilde en az 2 mm, optimal şartlarda 3-4 mm olmasını önerir. ${ }^{[15,16]}$ Vanlommel ve ark., hem tibial baseplate hem de kemiğe birer kat çimento sürerek 3-5 mm'lik bir çimentolama derinliği elde ettiklerini bildirmiştir. ${ }^{[17]}$ Sklerotik kemiğin varlığında penetrasyonu sağlamak için ek deliklerin drillenmesi implantın sağlıklı bir şekilde oturması ve lift-offu (implantın kalkmasını) önlemek için güzel bir tekniktir. Daha fazla derinliğe çimento yerleştirilirse termal nekroza sebep olacağı için dikkatli olunmalıdır.

Diz protezlerinin uzun sağkalımı için doğru dizilim esastır. Cerrahi sonrası bozuk dizilimi olan dizlerde, asimetrik yüklenmeye bağlı olarak, aseptik gevşeme ve osteoliz daha kolay meydana gelir. ${ }^{[18,19]}$ Bazı yazarlar, dizilim bozukluğunun, aseptik gevşeme için en önemli risk faktörü olduğunu bildirmişlerdir. Kim ve ark.'nın geniş katılımlı bir kohort çalışmasında, tibial implantın varusda olmasının yanı sıra $3^{\circ}$ 'den küçük ve $7^{\circ}$ 'den büyük tibial eğimin (slope) aseptik gevşemeyle ilişkisi olduğu bildirilmiştir. Aynı çalışmaya göre, femoral komponentin gevşemesi, tibial komponentin gevşemesine ikincil olarak meydana geldiği gösterilmiştir. ${ }^{[18]}$ Lee ve ark. ise, femoral komponentin varusta yerleştirilmesinin tek başına aseptik gevşeme için risk faktörü olduğunu göstermişlerdir. Aynı çalışmada tibial komponentin mediale $4 \mathrm{~mm}$ çökmesi veya $2^{\circ}$ 'den fazla varusun oluşması ile aseptik gevşemenin daha sık meydana geldiği tespit edilmiştir. ${ }^{[19]}$

\section{TANI}

Aseptik gevşemenin tanısı her zaman klinikle uyumluluk göstermeyebilir. Ayrıntılı bir anamnez ve fizik muayene ile elde edilen bilgiler her zaman radyolojik tetkiklerle uyumluluk göstermediği için aseptik gevşeme dikkatlice değerlendirilmesi gereken bir durumdur. ${ }^{[20]}$

Aşınmanın ve erken dönemde aseptik gevşemenin semptomatik bir kliniği yoktur. İkincil olarak meydana gelen osteoliz ve geç dönemdeki metallozise bağlı kronik sinovit başlar (Şekil 1). Bununla birlikte ağrılar ve efüzyonlar görülür. Hastalar genelde hareket
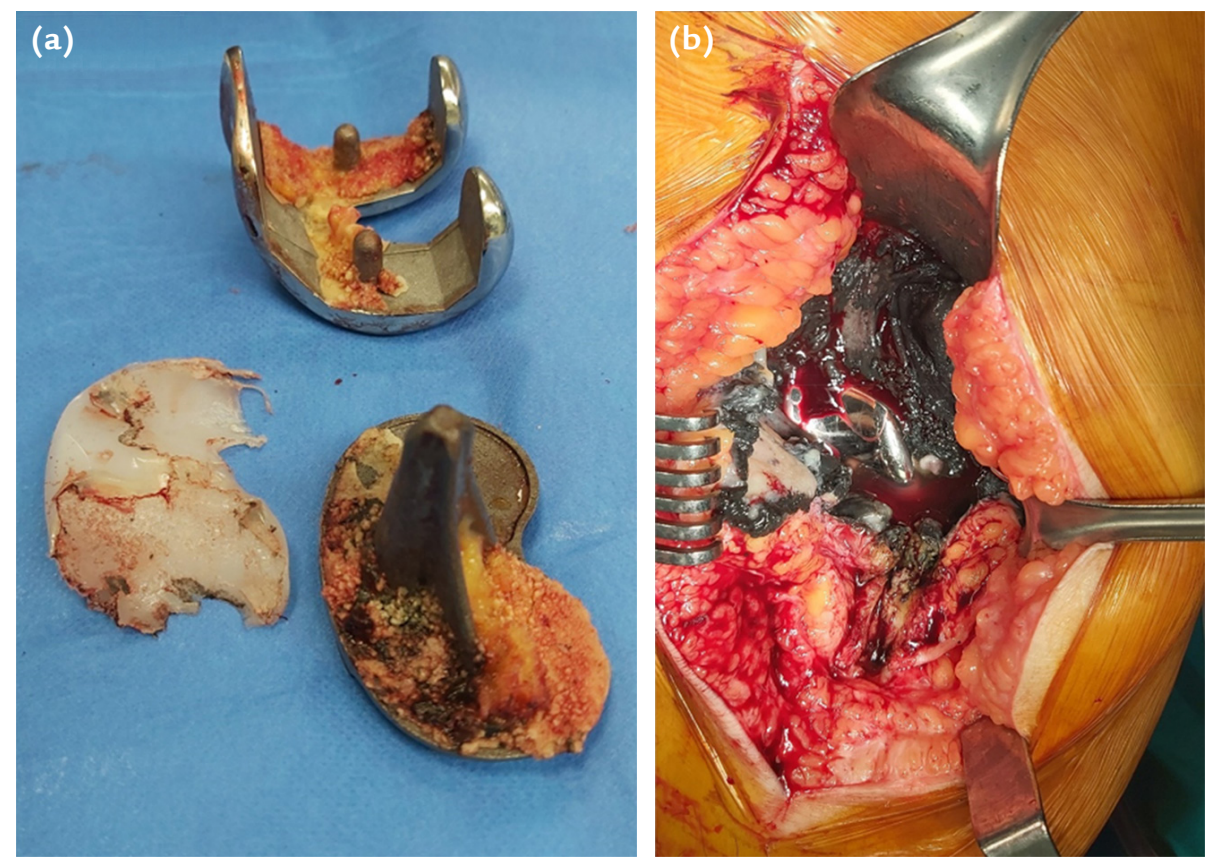

Şekil 1. a, b. Polietilen aşınması nedeniyle ameliyat edilen hastadan çıkan implantlar: Polietilen ara yüzeyinde meydana gelmiş aşınma görünüyor (a). Bu derece bir aşınma eklemde yaygın enflamasyon ve metallozise neden olmuş (b). 

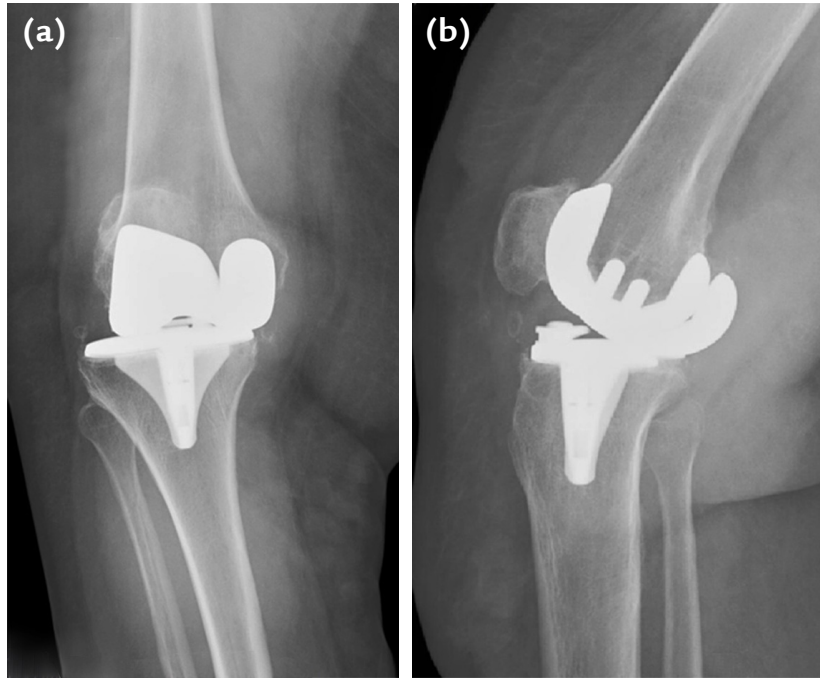

Şekil 2. a, b. Daha önce total diz artroplastisi uygulanmış hastanın medial kompartman aralığı daralmış olup aşınmayı düşündürüyor. Bu hastalara anestezi altında mutlaka yan (kollateral) bağ muayenesi yapılmalıdır; ön-arka (a) ve yan (b) grafiler.
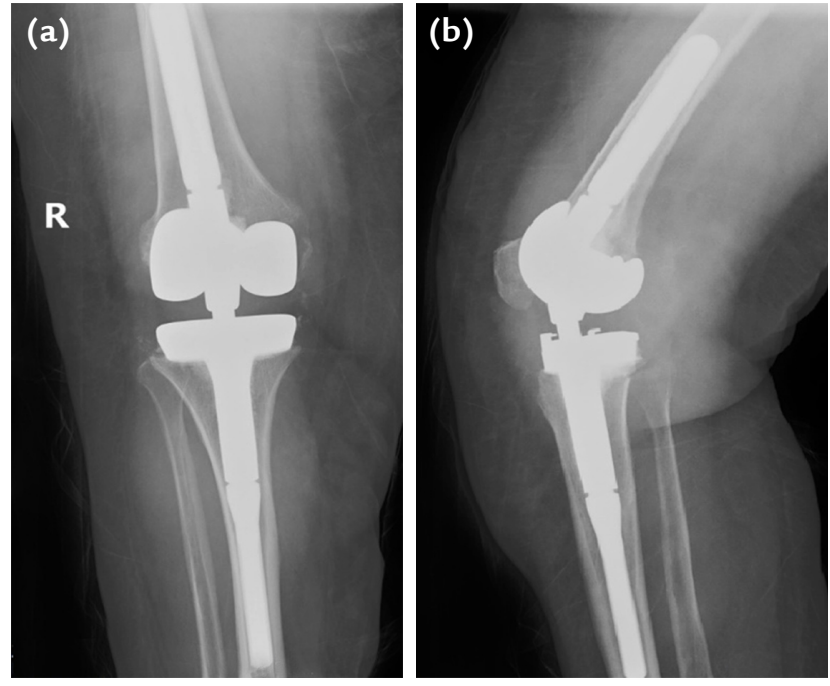

Şekil 3. a, b. Aynı hastaya, yaygın debridman sonrası, bağ hasarı nedeniyle menteşeli bir implant ile revizyon cerrahisi uygulandı; revizyon sonrası ön-arka (a) ve yan (b) grafiler.

son yıllarda kullanımı gittikçe artan, tek foton emisyon bilgisayarlı tomografinin (SPECT/CT) aseptik gevşeme için özgüllüğü ve duyarlılığı yüksek olan bir tetkik olduğu gösterilmiştir. ${ }^{[20,21]}$

\section{TEDAVi}

Asemptomatik hastalarda aseptik gevşemenin tedavisi konservatiftir. Hastalar sık sık takibe çağrılmaIı ve gerekli radyolojik analizler yapılmalıdır. Tedavi aşınma miktarına, implantın stabilitesine ve hastanın şikâyetine göre şekillenir.

Aşınma sadece PE ile sınırlı olup erken teşhis edilirse, basit bir PE değiştirilmesiyle tedavi edilebilir. Metale kadar inen bir aşınma söz konusu ise, tek komponent veya total eklem revizyonu düşünülür (Şekil 3). Aşınma, dizilim bozukluğuna ve bağ instabilitesine bağlı ise erken dönemde protez revizyonu ile tedavi gerekebilir. Doğru dizilim ve bağ dengesi sağlanmadığı sürece tekrardan aşınma meydana geleceği için revizyon cerrahisi kaçınılmazdır. ${ }^{[6,10,12]}$

Osteoliz nedeniyle, gevşemiş implantların altında sıklıkla kemik defektlerine rastlanır. Bu defektleri revizyon cerrahisi esnasında doldurmak önemlidir. Genç hastalarda, kemik stoğunu koruma amacıyla kemik greftleri tercih edilirken, daha yaşlı hastalarda prostetik metal destekler (augmentler) tercih edilebilir. On santimetreden küçük defektler için ise kemik çimentosu iyi bir alternatiftir. ${ }^{[22,23]}$ 


\section{KAYNAKLAR}

1. Hampton CB, Berliner ZP, Nguyen JT, Mendez L, Smith SS, Joseph AD, Padgett DE, Rodriguez JA. Aseptic Loosening at the Tibia in Total Knee Arthroplasty: A Function of Cement Mantle Quality? J Arthroplasty 2020;35(6):S190-6. Crossref

2. Sharkey PF, Lichstein PM, Shen C, Tokarski AT, Parvizi J. Why are total knee arthroplasties failing today -has anything changed after 10 years? J Arthroplasty 2014;29(9):1774-8. Crossref

3. Azar F, Canale T, Beaty J. Reconstructive procedures of the knee in adults. In: Azar F, editor. Campbell's Operative Orthopaedics, 13th ed. Canada: Elsevier; 2016. p.456-7.

4. Massin P. How does total knee replacement technique influence polyethylene wear? Orthop Traumatol Surg Res 2017;103(1):S21-7. Crossref

5. Panin SV, Kornienko LA, Huang Q, Buslovich DG, Bochkareva SA, Alexenko VO, Panov IL, Berto F. Effect of adhesion on mechanical and tribological properties of glass fiber composites, based on ultra-high molecular weight polyethylene powders with various initial particle sizes. Materials 2020;13(7):1602. Crossref

6. Goodman SB, Gallo J. Periprosthetic osteolysis: mechanisms, prevention and treatment. J Clin Med 2019;8(12):2091. Crossref

7. Lei P-F, Hu R-Y, Hu Y-H. Bone defects in revision total knee arthroplasty and management. Orthop Surg 2019;11(1):1524. Crossref

8. Pitta M, Esposito Cl, Li Z, Lee Y-Y, Wright TM, Padgett DE. Failure after modern total knee arthroplasty: a prospective study of 18,065 knees. J Arthroplasty 2018;33(2):407-14. Crossref

9. Garceau SP, Harris NH, Felberbaum DL, Teo GM, Weinblatt Al, Long WJ. Reduced Aseptic Loosening With Fully Cemented Short-Stemmed Tibial Components in Primary Cemented Total Knee Arthroplasty. J Arthroplasty 2020;35(6):1591-4. e3. Crossref

10. Apostu D, Lucaciu O, Berce C, Lucaciu D, Cosma D. Current methods of preventing aseptic loosening and improving osseointegration of titanium implants in cementless total hip arthroplasty: a review. J Int Med Res 2018;46(6):2104-19. Crossref

11. Koks S, Wood DJ, Reimann E, Awiszus F, Lohmann $\mathrm{CH}$, Bertrand J, Prans E, Maasalu K, Märtson A. The genetic variations associated with time to aseptic loosening after total joint arthroplasty. J Arthroplasty 2020;35(4):981-8. Crossref

12. Miller AJ, Stimac JD, Smith LS, Feher AW, Yakkanti MR, Malkani AL. Results of cemented vs cementless primary total knee arthroplasty using the same implant design. J Arthroplasty 2018;33(4):1089-93. Crossref
13. Goodnough LH, Finlay AK, Huddleston III JI, Goodman SB, Maloney WJ, Amanatullah DF. Obesity is independently associated with early aseptic loosening in primary total hip arthroplasty. J Arthroplasty 2018;33(3):882-6. Crossref

14. Gothesen O, Lygre SHL, Lorimer M, Graves S, Furnes O. Increased risk of aseptic loosening for 43, 525 rotatingplatform vs. fixed-bearing total knee replacements: A Norwegian-Australian registry study, 2003-2014. Acta Orthop 2017;88(6):649-56. Crossref

15. Schwarze M, Schonhoff $M$, Beckmann NA, Eckert J, Bitsch $\mathrm{R}$, Jäger S. Femoral cementation in knee arthroplasty -a comparison of three cementing techniques in a sawbone model using the ATTUNE knee. J Knee Surg 2021;34(3):25866. Crossref

16. Zhou K, Yu H, Li J, Wang H, Zhou Z, Pei F. No difference in implant survivorship and clinical outcomes between fullcementless and full-cemented fixation in primary total knee arthroplasty: a systematic review and meta-analysis. Int J Surg 2018;53:312-9. Crossref

17. Vanlommel J, Luyckx JP, Labey L, Innocenti B, De Corte R, Bellemans J. Cementing the tibial component in total knee arthroplasty: which technique is the best? J Arthroplasty 2011;26(3):492-6. Crossref

18. Kim Y-H, ParkJ-W, Kim J-S, Park S-D. The relationship between the survival of total knee arthroplasty and postoperative coronal, sagittal and rotational alignment of knee prosthesis. Int Orthop 2014;38(2):379-85. Crossref

19. Lee B-S, Cho H-I, Bin S-I, Kim J-M, Jo B-K. Femoral component varus malposition is associated with tibial aseptic loosening after TKA. Clinical Orthop Relat Res 2018;476(2):400-7. Crossref

20. Barnsley L, Barnsley L. Detection of aseptic loosening in total knee replacements: a systematic review and meta-analysis. Skeletal Radiol 2019;48(10):1565-72. Crossref

21. Schwaiger BJ, Gassert FT, Suren C, Gersing AS, Haller B, Pfeiffer D, Dangelmaier-Dawirs J, Roski F, von EisenhartRothe R, Prodinger PM, Woertler K. Diagnostic accuracy of MRI with metal artifact reduction for the detection of periprosthetic joint infection and aseptic loosening of total hip arthroplasty. Eur J Radiol 2020;131:109253. Crossref

22. Hinman AD, Prentice HA, Paxton EW, Kelly MP. Modular Tibial stem use and risk of revision for aseptic loosening in cemented primary Total knee Arthroplasty. J Arthroplasty 2021;36(5):1577-83. Crossref

23. Levent A, Suero EM, Gehrke T, Bakhtiari IG, Citak M. Risk factors for aseptic loosening in complex revision total knee arthroplasty using rotating hinge implants. Int Orthop 2021;45(1):125-32. Crossref 